\title{
Estructura y composición arbórea en un gradiente altitudinal del Área Natural Protegida Metzabok, Chiapas, México
}

Facundo Sánchez Gutiérrez ${ }^{1,2}$, Juan Ignacio Valdez Hernández², Patricia Hernández de la Rosa², Julio Sánchez Escudero ${ }^{2}$, Ángel Sol Sánchez ${ }^{3 *}$, Carlos Castillejos Cruz ${ }^{4}$

\& Alfredo Isaac Brindis-Santos ${ }^{5}$

1. Facultad Maya de Estudios Agropecuarios, Universidad Autónoma de Chiapas, Carretera Catazajá-Palenque km 4, C.P. 29980, Catazajá, Chiapas; agrofor03@gmail.com

2. Colegio de Postgraduados, Campus Montecillo, km 36.5 Carretera México-Texcoco. C. P. 56230. Montecillo, Texcoco, Estado de México; ignaciov@colpos.mx, pathr@colpos.mx, clarijul@hotmail.com

3. Colegio de Postgraduados, Campus Tabasco, Periférico Carlos A. Molina S/N, km 3.5, Cárdenas, Tabasco, México. C. P. 86500; sol@colpos.mx

4. Universidad Autónoma de México, Avenida Guelatao No. 66 El Paraíso Iztapalapa, Distrito Federal Campus I, Facultad de Estudios Superiores Zaragoza; carlcasti@colpos.mx

5. Facultad de Ciencias Agrícolas, Campus IV, Universidad Autónoma de Chiapas, Entronque Carretera Costera y Huehuetán Pueblo, C.P. 30660. Huehuetán, Chiapas; brin-dis@hotmail.com

* Correspondencia

Recibido 14-II-2020. Corregido 15-VI-2020. Aceptado 29-IX-2020.

\begin{abstract}
Tree structure and composition in an altitude gradient of the Metzabok Protected Natural Area, Chiapas, Mexico. Introduction: The forests are home to more than half of the planet's species, despite of their biodiversity, they are the ecosystems most threatened mainly by anthropic activities, but their complexity in relation to altitude has information gaps despite of the values it represents. Objective: Describe the structure and diversity of trees on an altitudinal gradient. Methods: In five altitudinal strata (EA) 21 sampling plots (UM) of $20 \times 50 \mathrm{~m}$ were established: six in EA1, five in EA2, four in EA3, three in EA4 and 5. The structure was characterized by the categories diametric and height, and the importance value indexes (IVI) and forestry (IVF). Diversity was analyzed with the Shannon-Wiener $\left(\mathrm{H}^{\prime}\right)$ and Simpson $(\mathrm{S})$ indices, while the floristic similarity with the Sørensen coefficient (IS). Results: It was registered 209 tree species, eight are listed in the Official Mexican Standard-059-Secretariat of the Environment and Natural Resources-2010 (NOM059-SEMARNAT-2019) as vulnerable species. The species with the highest IVI and IVF were Haematoxylum campechianum and Manilkara zapota in EA1; Rinorea guatemalensis and Pouteria reticulata in EA2; M. zapota and Brosimum alicastrum at EA3; Guatteria anomala and Pseudolmedia spuria in EA4; and Terminalia amazonia and Pouteria durlandii at EA5. In the five EAs, three ranges of height and six of diameter were identified, the largest number of individuals was found in the first diametric range. Species diversity was lower at higher altitudes. In EA 2 and 3 the greatest similarity was presented (IS= $58.7 \%$ ). Conclusion: The structure and diversity of the tree species present greater changes in wider altitude ranges, related to the environmental need of each species.
\end{abstract}

Key words: elevation; ecological structure; basal area; species richness; similarity.

Sánchez Gutiérrez, F., Valdez Hernández, J.I., Hernández de la Rosa, P., Sánchez Escudero, J., Sol Sánchez, A., Castillejos Cruz, C., \& Brindis-Santos, A.I. (2021). Estructura y composición arbórea en un gradiente altitudinal del Área Natural Protegida Metzabok, Chiapas, México. Revista de Biología Tropical, 69(1), 12-22. DOI 10.15517/rbt.v69i1.40689 
En la zona intertropical se encuentran las comunidades vegetales terrestres más antiguas en el mundo como las selvas tropicales húmeda y seca (Koleff, Urquiza-Hass, \& Contreras, 2012), ecosistemas ricos, diversos y complejos (Pennington \& Sarukhán, 2005) que cubren cerca del $7 \%$ de la superficie terrestre con 1 172 millones de hectáreas (Sánchez-Gutiérrez, Valenzuela-Gómez, Valdez-Hernández, \& González-González, 2017). De ellas, 656 millones de ha (56 \%) se encuentran en América (Dirzo, Aguirre, \& López, 2009); 32.1 millones de ha (4.9\%) en México (CONABIO, 2008) y 1.3 millones ( $3 \%$ ) de ha en el estado de Chiapas (Cruz-Lara, Lorenzo, Soto, Naranjo, \& Ramírez, 2004). Mientras tanto, con solo el $0.16 \%$ del territorio del país, la selva lacandona alberga más del $20 \%$ del total de las especies existentes en México (Durán-Fernández, Aguirre-Rivera, García-Pérez, Levy-Tacher, \& Nova-Vázquez, 2016).

Aunque las selvas del neotrópico poseen gran biodiversidad son los ecosistemas más amenazados, principalmente por la deforestación, extracción de madera, caza, urbanización y aperturas de vías de comunicación; además de eventos naturales como huracanes, incendios, plagas y enfermedades (SánchezGutiérrez et al., 2017). En los últimos 150 años se ha perdido más del $30 \%$ de su superficie a nivel mundial (ter Steege et al., 2015), debido a la falta de la aplicación de las políticas públicas relacionadas con el cambio y uso de suelo, causando modificaciones en la estructura y composición florística de los reductos de selvas (Sánchez-Gutiérrez, Valdez-Hernández, Hernández-de la Rosa, \& Beltrán-Rodríguez, 2019), así como en su composición de especies de las comunidades arbóreas a través de la reducción del hábitat causado por las actividades antropogénicas (González \& Hamrick, 2005). Además, la fragmentación provoca que se reduzca la mayoría de las plantas del sotobosque en la selva tolerante a la sombra, al tiempo que se fomenta la presencia de especies de plantas generalistas y pioneras (Clark \& Clark, 1987). Por lo tanto, cualquier alteración en las características del dosel, pone en riesgo al sotobosque para mantener la estructura, el funcionamiento y la dinámica de la selva (Brienen \& Zuidema, 2006).

Los estudios sobre la estructura y diversidad arbórea en las selvas y bosques, proporcionan información botánica y ecológica con respecto a su dinámica y favorecen la definición de estrategias para el manejo sustentable (Basáñez, Alanís, \& Badillo, 2008). El análisis de los cambios en la vegetación en gradientes altitudinales, contribuyen a determinar los patrones de distribución de especies arbóreas en condiciones ambientales diversas y en extensiones geográficas amplias, además de conocer como el cambio climático (CC) influye en la migración de las especies (Mateo, Felicísimo, \& Muñoz, 2011). En este sentido Guariguata, Cornelius, Locatelli, Forner y Sánchez-Azofeifa (2008) proponen el manejo de las selvas, con base en aprovechamiento selectivo, manteniendo especies que pueden adaptarse a las variaciones climáticas. A su vez Grubb (1977) argumenta que los gradientes altitudinales están relacionados con la composición y distribución de las especies, debido a factores como el relieve, temperatura, precipitación. Además la nubosidad, limita la radiación fotosintéticamente activa disponible, responsables de la productividad de las selvas (Grubb, 1977).

La Selva Lacandona es considerada uno de los últimos reductos de selvas del trópico húmedo de México. A pesar de la diversidad de especies de flora y fauna que alberga son escasos los estudios, principalmente los que describen la estructura y diversidad de las especies arbóreas y los cambios en estos atributos con relación a la altitud (Sánchez-Gutiérrez et al., 2019). Por lo expuesto, el presente estudio tuvo como objetivo principal describir la estructura y la composición de las especies arbóreas en un gradiente altitudinal en el área natural protegida (ANP) Metzabok, Selva Lacandona, Chiapas.

\section{MATERIALES Y MÉTODOS}

Área de estudio: Se localiza en el área natural protegida (ANP) Metzabok, la cual 
se ubica al noreste de la Selva Lacandona en la comunidad de Puerto Bello Metzabok, Ocosingo, Chiapas, México, entre las coordenadas geográficas $\left(17^{\circ} 08^{\prime} 36^{\prime \prime} \mathrm{N} \& 91^{\circ} 34^{\prime} 42^{\prime \prime} \mathrm{W}\right)$, en una superficie de 3368 ha. El clima se caracteriza por ser cálido húmedo con lluvias todo el año Af (m), la temperatura media anual es de $24{ }^{\circ} \mathrm{C}$ y precipitación total anual de 3160 $\mathrm{mm}$. Los tipos de suelo son redzinas, luvisoles, gleysoles, vertisoles, cambisoles y litosoles, la vegetación predominante es selva alta, mediana perennifolia, subperennifolia y vegetación secundaria (Sánchez-Gutiérrez et al., 2017).

Muestreo y medición de variables: Con base en el mapa de curvas de nivel se determinaron cinco estratos altitudinales (EA) en intervalos de $100 \mathrm{~m}$; en ellos se instalaron aleatoriamente 21 unidades de muestreo (UM) de $20 \times 50 \mathrm{~m}\left(1000 \mathrm{~m}^{2}\right)$ : seis en el estrato altitudinal 1 (EA1), cinco en el EA2, cuatro en el
EA3, tres en el EA4 y tres en el EA5 (Fig. 1). Cada UM fue subdividida en 10 subunidades de muestreo (SUM) de $10 \times 10 \mathrm{~m}$.

Composición de especies arbóreas: A los individuos con diámetro $\geq 2.5 \mathrm{~cm}$ se les midió su diámetro normal $(\mathrm{DN})$, altura total $(\mathrm{Ht})$ y el diámetro de copa (menor y mayor) (CarreónSantos \& Valdez-Hernández 2014). La identificación taxonómica de las especies arbóreas se realizó con ayuda de manual y guía de campo (Pennington \& Sarukhán, 2005). De los árboles desconocidos se recolecto material botánico que se herborizó e identificó en el herbario ECOSC-H del ECOSUR en San Cristóbal de las Casas, Chiapas. Los nombres científicos se verificaron en el sitio web theplantlist.org/. Se elaboraron curvas especies-área para los cinco EA, que representan gráficamente la riqueza acumulada de especies en una superficie determinada (Beltrán-Rodríguez et al., 2018).

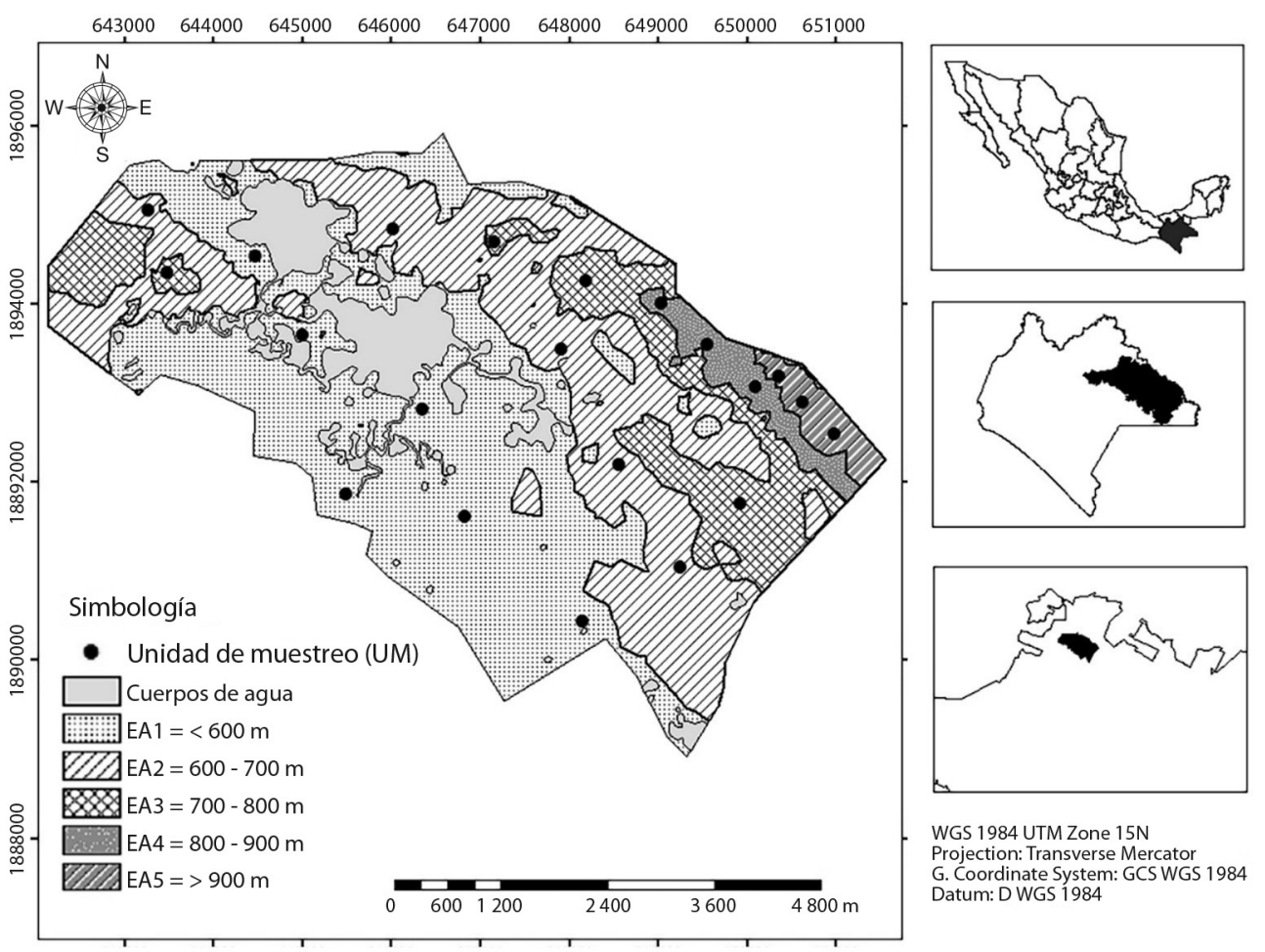

Fig. 1. Distribución de las unidades de muestreo (UM) en cinco estratos altitudinales.

Fig. 1. Distribution of sampling plots (UM) in five altitudinal strata. 
Análisis de la estructura arbórea: $\mathrm{La}$ categoría de altura (individuos con $\mathrm{DN} \geq 2.5$ $\mathrm{cm})$ se determinó mediante las inflexiones de curvas generadas al graficar el número de individuos contra su altura (López-Toledo, ValdezHernández, Pérez-Farrera, \& Cetina-Alcalá, 2012). Para las diamétricas se establecieron en intervalos de $10 \mathrm{~cm}$ de amplitud para cada EA. Para jerarquizar la dominancia de cada especie se calculó el índice de valor de importancia (IVI) (Zarco et al., 2010) con la ecuación: ; donde: $D r=$ Densidad relativa; $A B r=$ Área basal relativa; $\mathrm{Fr}=$ Frecuencia relativa. También se determinó el índice de valor forestal (IVF), que incluye la variable vertical de altura (Carreón-Santos \& Valdez-Hernández, 2014) con la ecuación: $I V F=D N r+A r+C r$; donde: $D N r=$ Diámetro normal relativo; $A r=$ Altura total relativa; $\mathrm{Cr}=$ Cobertura de copa relativa.

Diversidad de especies arbóreas: Se calcularon los índices de Shannon-Wiener $\left(H^{\prime}\right)$, alfa de Fisher $(\alpha)$, que en todos los casos cumplió con la restricción para su uso de $\mathrm{N} / \mathrm{S} \geq 1.4$; en donde $\mathrm{N}$ es el número total de observaciones y $\mathrm{S}$ es la riqueza e índice de Simpson $(S)$ (Zarco et al., 2010).

Se utilizó la prueba de "t" modificada por Hutcheson (Carreón-Santos \& Valdez-Hernández, 2014), para verificar diferencias estadísticamente significativas en el índice de Shannon-Wiener; los demás índices de diversidad y los valores de área basal, altura y densidad fueron comparados entre cada estrato mediante un análisis de varianza (ANOVA) y prueba de Tukey $(\mathrm{P} \leq 0.05)$ con el software SAS 9.4.

Semejanza florística entre los estratos altitudinales: Se utilizó el índice de Sørensen, que se basa en la presencia-ausencia de especies entre dos comunidades (Sánchez-Gutiérrez et al., 2017). La ecuación utilizada fue:

$$
I S=\left[\frac{2 C}{A+B} X 100\right]
$$

donde: $A=$ número de especies en la comunidad $1 ; B=$ número de especies en la comunidad 2; $C=$ número de especies comunes en ambas comunidades.

\section{RESULTADOS}

Composición florística arbórea: Se registraron 4584 individuos de 65 familias, 155 géneros y 209 especies arbóreas. De las especies registradas, ocho se encuentran enlistadas en la NOM-059-SEMARNAT-2019 como vulnerables; cinco como amenazadas: Astronium graveolens Jacq., Calophyllum brasiliense Cambess, Geonoma pinnatifrons Willd., Guatteria anomala R.E. Fr. y Magnolia lacandoni$c a$; dos en peligro de extinción: Louteridium donnell-smithii S. Watson y Vatairea lundellii (Standl.) Record; y una en protección especial: Cedrela odorata L.

En el EA1, las UM instaladas cerca de las lagunas $(100$ a $500 \mathrm{~m})$ registró alta frecuencia de Haematoxylum campechianum L. y Pachira aquatica Aubl. Las UM situadas en áreas perturbadas por la deforestación e incendio en 1983, presentaron a Heliocarpus appendiculatus Turcz., y Bursera simaruba (L.) Sarg., como las especies dominantes. En los EA2 y 3 las especies comunes fueron: Pouteria reticulata (Engl.) Eyma, Pseudolmedia glabrata (Liebm.) C.C.Berg, Quararibea funebris (La Llave) Vischer y Brosimum alicastrum Sw. y en los EA4 y 5 fueron: Pouteria durlandii (Standl.) Baehni, Dialium guianense (Aubl.) Sandwith, Pseudolmedia spuria (Sw.) Griseb. y Guarea glabra Vahl.

La curva de acumulación de especies muestra que ninguno de los EA analizados alcanzó el nivel de la asíntota (Fig. 2). Por lo tanto, se necesita un mayor esfuerzo de muestreo para registrar la mayoría de las especies.

Estructura vertical: En los cinco estratos se registraron tres rangos de altura (baja, media y alta). La baja con altura promedio de $5 \pm 3 \mathrm{~m}$, las especies más frecuentes fueron: Myriocarpa longipes Liebm, Piper psilorhachis C. DC., Randia aculeata L. y Trichilia pallida Sw; media con altura promedio de $17 \pm 5 \mathrm{~m}$, dominaron Garcinia intermedia (Pittier) Hammel, 


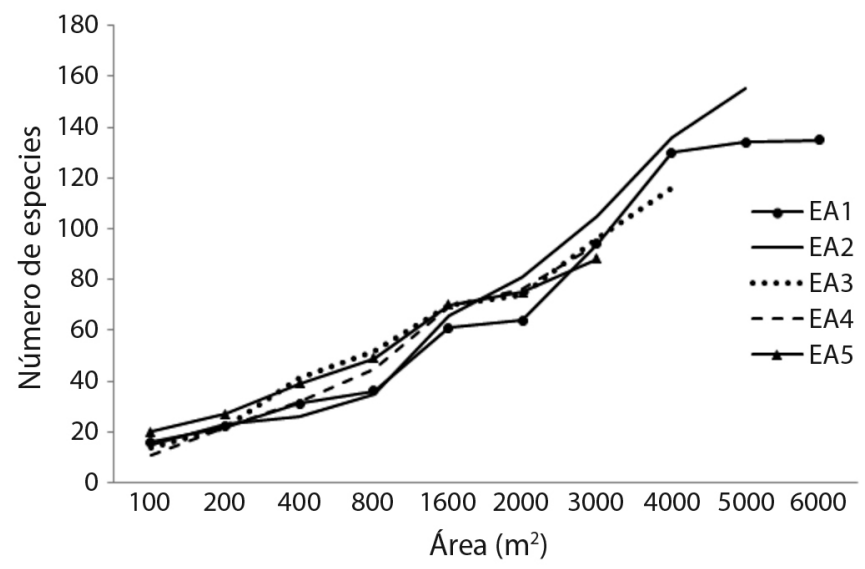

Fig. 2. Curva especies-área con cinco estratos altitudinales (EA).

Fig. 2. Species-area curve with five altitudinal strata (EA).

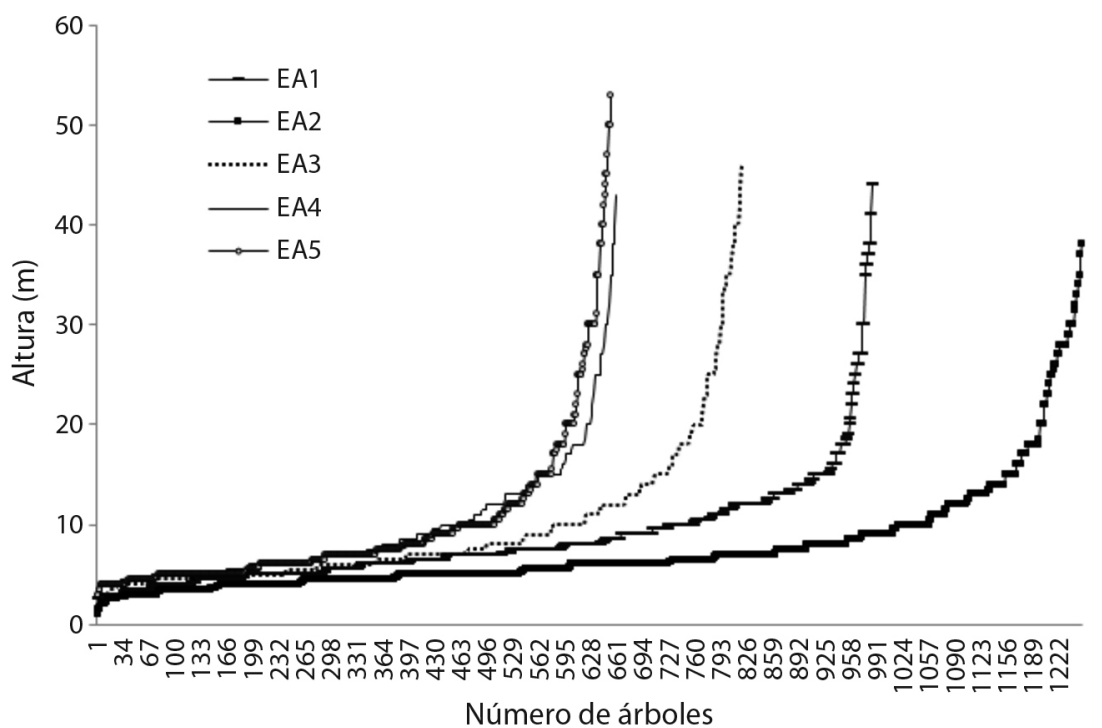

Fig. 3. Altura de árboles en cinco estratos altitudinales (EA).

Fig. 3. Tree height in five altitudinal strata (EA).

Pseudolmedia spuria (Sw.) Griseb., Pseudolmedia glabrata (Liebm.) C.C. Berg, Pouteria multiflora (A. DC.) Eyma y Quararibea funebris (La Llave) Vischer; y alta con altura promedio de $17 \pm 42 \mathrm{~m}$, fue representado por Calophyllum brasiliense Cambess., Dialium guianense (Aubl.) Sandwith, Guatteria anomala R.E.Fr., Manilkara chicle (Pittier) Gilly, Manilkara zapota (L.) P. Royen y Terminalia amazonia (J.F. Gmel.) Exell (Fig. 3). Los EA1 y 2 mostraron diferencias estadísticas significativas (Tukey, $\mathrm{P}<0.05$ ) en altura con los EA3, 4 y 5 , registrando árboles de hasta $53 \mathrm{~m}$ de altura.

Distribución horizontal: La densidad de individuos fue mayor en el EA2 con 2504 ind. $\mathrm{ha}^{-1}$, y menor en el EA1 con 1998 ind. ha ${ }^{-1} \mathrm{y}$ no hubo un patrón de distribución en relación 


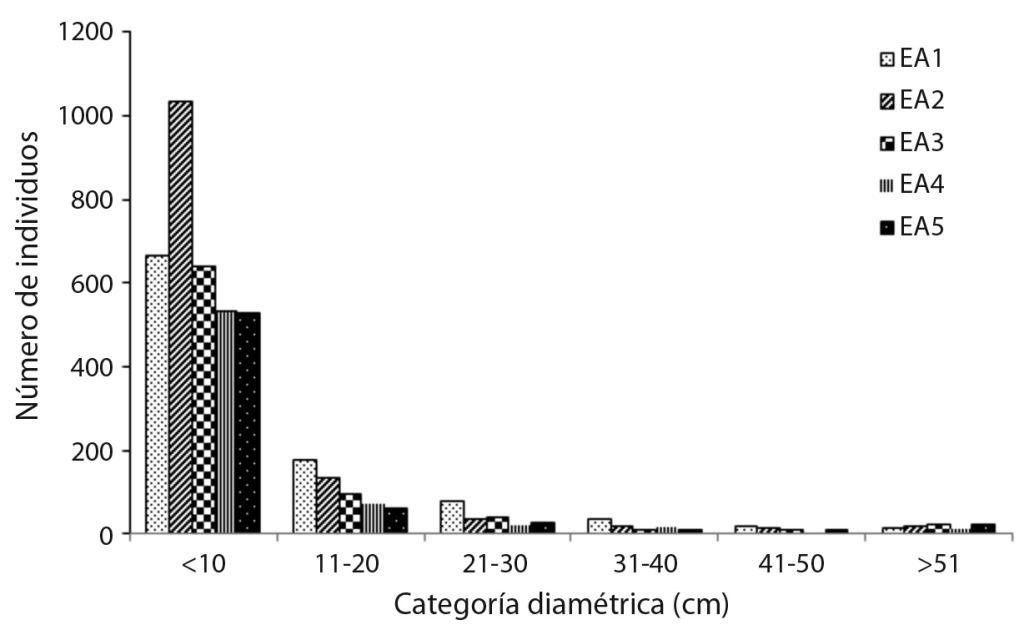

Fig. 4. Distribución diamétrica de árboles en cinco estratos altitudinales (EA).

Fig. 4. Diametric distribution of trees in five altitudinal strata (EA).

con la altitud. El diámetro presenta diferencias significativas (Tukey, $\mathrm{P}<0.05$ ) entre $\mathrm{EA}$, con una escala de 2.5 a $199 \mathrm{~cm}$ y una media de 9.7 $\mathrm{cm}$. El área basal registró valor más alto en el EA5 con $80.8 \mathrm{~m}^{2} \mathrm{ha}^{-1} \mathrm{y}$ menor en el EA2 con $35.6 \mathrm{~m}^{2} \mathrm{ha}^{-1}$. En todos los EA se obtuvieron un mayor número de individuos en la primera categoría diamétrica con una disminución progresiva en las siguientes mostrando una distribución de "J" invertida (Fig. 4).

Índice de valor de importancia (IVI) y forestal (IVF): Las especies con los valores estructurales más altos fueron: $H$. campechianum, M. zapota para el EA1, Rinorea guatemalensis (S. Watson) Bartlett, P. reticulata y $B$. alicastrum para el EA2, M. zapota, B. alicastrum, $R$. guatemalensis en el EA3, $G$. anomala, P. spuria y P. durlandii en EA4 y $T$. amazonia, $P$. durlandii y D. guianense para el EA5 (Tabla 1).

Diversidad de especies arbóreas: Existe diferencias significativas $(\mathrm{P}<0.05)$ entre la diversidad de los EA, de acuerdo con la prueba de la t modificada de Hutcheson, corroborado con los valores de índices de Shannon. El mayor valor se presenta en el EA1 $\left(H^{\prime}=3.7\right)$ y el menor en el EA5 $\left(H^{\prime}=3.2\right)$ (Tabla 2$)$. Este orden coincide con el valor de Simpson e indica la dominancia de ciertas especies a una mayor altitud. Estos valores muestran que la selva del ANP Metzabok presenta una relación inversa, a mayor altitud menor diversidad de especies arbóreas. El mayor valor de alfa de Fisher se presenta en el EA2 (Tabla 2), debido a la mayor densidad de individuos en comparación con los otros EA.

Semejanza florística: Al comparar los valores del índice de Sørensen, los EA más semejantes fueron el 2 y 3 con un $I S=58.7 \%$ y el valor menor se presentó entre el EA1 y 5 con un $I S=39.5$. Todos los EA son florísticamente parecidos al compartir el $50 \%$ de las especies arbóreas.

\section{DISCUSIÓN}

El Área Natural Protegida Metzabok, registro una riqueza de 209 especies, valor superior comparando con otros trabajos realizados en selvas de la región tropical como las de China por Ding, Zang, Liu, He y Letche (2012) con 171 especies, de Australia por Bradford, Metcalfe, Ford, Liddell y Mckeown (2014) con 208 especies, de India por Sagar, Raghubanshi y Singh (2003) con 49 especies, así como de México por Zarco, Valdez-Hernández, 
TABLA 1

Especies arbóreas con mayores índices de valor de importancia (IVI) y de valor forestal (IVF)

TABLE 1

Tree species with higher indices of importance value (IVI) and forest value (IVF)

\begin{tabular}{|c|c|c|c|}
\hline Especie & IVI & Especie & IVF \\
\hline Estrato altitudinal $1(500-600 \mathrm{~m})$ & & Estrato altitudinal $1(500-600 \mathrm{~m})$ & \\
\hline Haematoxylum campechianum $\mathrm{L}$. & 66.4 & Haematoxylum campechianum $\mathrm{L}$. & 81.5 \\
\hline Manilkara zapota (L.) Royen & 16.5 & Manilkara zapota (L.) Royen & 14.4 \\
\hline Eugenia gaumeri Standl. & 12.7 & Heliocarpus appendiculatus Turcz. & 12.7 \\
\hline Hauya elegans DC. & 9.9 & Rinorea guatemalensis (S. Watson) Bartlett & 9.4 \\
\hline Pachira aquatica Aubl. & 8.9 & Bursera simaruba (L.) Sarg. & 8.0 \\
\hline 130 especies restantes & 185.6 & 130 especies restantes & 174.1 \\
\hline Estrato altitudinal $2(600-700 \mathrm{~m})$ & & Estrato altitudinal $2(600-700 \mathrm{~m})$ & \\
\hline Rinorea guatemalensis (S. Watson) Bartlett & 27 & Rinorea guatemalensis (S. Watson) Bartlett & 32.2 \\
\hline Pouteria reticulata (Engl.) Eyma & 15.2 & Pseudolmedia glabrata (Liebm.) C.C.Berg & 14.5 \\
\hline Brosimum alicastrum $\mathrm{Sw}$. & 14 & Pouteria reticulata (Engl.) Eyma & 14.4 \\
\hline Pouteria durlandii (Standl.) Baehni & 12.5 & Brosimum alicastrum $\mathrm{Sw}$. & 12.3 \\
\hline Manilkara zapota (L.) Royen & 10.9 & Dialium guianense (Aubl.) Sandwith & 10.8 \\
\hline 150 especies restantes & 220.4 & 150 especies restantes & 215.8 \\
\hline Estrato altitudinal $3(700-800 \mathrm{~m})$ & & Estrato altitudinal $3(700-800 \mathrm{~m})$ & \\
\hline Manilkara zapota (L.) Royen & 20.9 & Brosimum alicastrum $\mathrm{Sw}$. & 25.05 \\
\hline Brosimum alicastrum $\mathrm{Sw}$. & 19.2 & Rinorea guatemalensis (S. Watson) Bartlett & 21.67 \\
\hline Rinorea guatemalensis (S. Watson) Bartlett & 16.4 & Pouteria reticulata (Engl.) Eyma & 17.09 \\
\hline Dialium guianense (Aubl.) Sandwith & 13.5 & Manilkara zapota (L.) Royen & 15.44 \\
\hline Pouteria reticulata (Engl.) Eyma & 13 & Dialium guianense (Aubl.) Sandwith & 14.54 \\
\hline 111 especies restantes & 216.9 & 111 especies restantes & 206.2 \\
\hline Estrato altitudinal $4(800-900 \mathrm{~m})$ & & Estrato altitudinal $4(800-900 \mathrm{~m})$ & \\
\hline Guatteria anomala R.E. Fr. & 23.2 & Pseudolmedia spuria (Sw.) Griseb. & 25.7 \\
\hline Pseudolmedia spuria (Sw.) Griseb. & 20.1 & Pouteria durlandii (Standl.) Baehni & 25.5 \\
\hline Pouteria durlandii (Standl.) Baehni & 19.3 & Dialium guianense (Aubl.) Sandwith & 17.7 \\
\hline Dialium guianense (Aubl.) Sandwith & 14.7 & Rinorea guatemalensis (S. Watson) Bartlett & 17.1 \\
\hline Pouteria multiflora (A. DC.) Eyma & 13.2 & Lacistema aggregatum (P.J. Bergius) Rusby & 14.6 \\
\hline 89 especies restantes & 209.6 & 89 especies restantes & 199.4 \\
\hline Estrato altitudinal 5 (>900 m) & & Estrato altitudinal $5(>900 \mathrm{~m})$ & \\
\hline Terminalia amazonia (J.F. Gmel.) Exell & 46.2 & Terminalia amazonia (J.F. Gmel.) Exell & 31.1 \\
\hline Pouteria durlandii (Standl.) Baehni & 17.8 & Pouteria durlandii (Standl.) Baehni & 25.4 \\
\hline Dialium guianense (Aubl.) Sandwith & 17 & Dialium guianense (Aubl.) Sandwith & 25.2 \\
\hline Pseudolmedia spuria (Sw.) Griseb. & 14.9 & Pseudolmedia spuria (Sw.) Griseb. & 23.6 \\
\hline Manilkara zapota (L.) Royen & 12.6 & Saurauia sp. & 20.5 \\
\hline 83 especies restantes & 191.3 & 83 especies restantes & 191.3 \\
\hline
\end{tabular}

Ángeles-Pérez y Castillo-Castillo (2010) con 71 especies y López-Toledo et al. (2012) con 83 especies. Estos resultados demuestran que el área de estudio, se encuentra entre las selvas con mayor riqueza de especies arbóreas en el mundo, favorecido por la suma de factores ecológicos del lugar como, altitud, temperatura, precipitación y el estado de conservación además de su ubicación geográfica.

Las especies más abundantes en los cinco estratos estudiados, se comportaron de manera similar a los registros de Levy, Aguirre, García y Martínez (2006) y Meave, Romero-Romero, Valle-Doménch, Rincón-Gutiérrez, Martínez 
TABLA 2

Índices de diversidad, número de riqueza y familia, promedio de diámetro y altura

TABLE 2

Diversity indices, number of wealth and family, average diameter and height

\begin{tabular}{|c|c|c|c|c|c|}
\hline & \multicolumn{4}{|c|}{ Estrato altitudinal } & \multirow[b]{2}{*}{ EA5 } \\
\hline & EA1 & EA2 & EA3 & EA4 & \\
\hline $\mathrm{DN}$ & $10.95_{\mathrm{c}}$ & $8.32_{\mathrm{a}}$ & $9.75_{a b c}$ & $9.0_{\mathrm{ab}}$ & $10.61 \mathrm{bc}$ \\
\hline $\mathrm{Ht}$ & $7.8_{a}$ & $7.4_{a}$ & $9.2_{\mathrm{b}}$ & $9.4_{b}$ & $9.6_{b}$ \\
\hline $\mathrm{R}$ & $135 \mathrm{abc}$ & $155_{\mathrm{c}}$ & $116_{a b}$ & $96_{b}$ & 87 b \\
\hline $\mathrm{F}$ & $42_{a b}$ & $46_{a}$ & $41_{a b}$ & $37 \mathrm{ab}$ & $33_{b}$ \\
\hline $\mathrm{S}$ & $3.91_{\mathrm{a}}$ & $3.89_{a b}$ & $3.85_{a b}$ & $3.75_{\mathrm{ab}}$ & $3.72 \mathrm{c}$ \\
\hline A & $39.06 \mathrm{a}$ & $46.15_{b}$ & $36.44_{a}$ & $29.97 \mathrm{ab}$ & $27.38 \mathrm{ab}$ \\
\hline $\mathrm{H}^{\prime}$ & $3.71_{\mathrm{a}}$ & $3.37_{\mathrm{ab}}$ & $3.36_{\mathrm{ab}}$ & $3.30_{\mathrm{ab}}$ & $3.27 \mathrm{~b}$ \\
\hline
\end{tabular}

$\mathrm{DN}=$ diámetro normal, $\mathrm{Ht}=$ altura total, $\mathrm{R}=$ riqueza de especies, $\mathrm{F}=$ Familia, $\mathrm{S}=$ índice de Simpson, $\alpha=$ alfa de Fisher, $\mathrm{H}^{\prime}$ $=$ índice de Shannon. Letras diferentes significa que hay diferencias significativas $(\mathrm{P}<0.05)$ entre los estratos altitudinales. $\mathrm{DN}=$ normal diameter, $\mathrm{Ht}=$ total height, $\mathrm{R}=$ species richness, $\mathrm{F}=$ Family, $\mathrm{S}=$ Simpson Index, $\alpha=$ a Fisher alpha, $\mathrm{H}^{\prime}=$ Shannon Index. Different letters mean significant differences $(\mathrm{P}<0.05)$ between altitudinal strata.

y Ramos (2008), reconocidas como parte de la selva alta perennifolia de México (Pennington \& Sarukhán, 2005). Además de la R. guatemalensis que coloniza los claros de las selvas, causados por la caída de las ramas o de árboles completos (Zarco et al., 2010; Vázquez-Negrín, Castillo-Acosta, Valdez-Hernández, ZavalaCruz, \& Martínez-Sánchez, 2011).

En el EA1, las UM instaladas cerca de las lagunas registraron alta frecuencia de $H$. campechianum y $P$. aquatica, especies que habitan en planicies y soportan inundación hasta seis meses al año (Tun-Dzul, Vester, \& Schmook, 2008). Las UM situadas en áreas perturbadas presentaron a $H$. appendiculatus y B. simaruba, consideradas como pioneras (Vázquez-Negrín et al., 2011) y comunes de la vegetación secundaria (Carreón-Santos \& Valdez-Hernández, 2014).

Los géneros Dialium, Pouteria, Pseudolmedia y Guarea, fueron registrados también en la selva amazónica de Bolivia por AraujoMurakami et al. (2015), Terminalia en Nigeria por Gourlet-Fleury et al. (2013), Randia, Bauhinia, Casearia y Mimosa en la India por Sagar et al. (2003). La presencia de estos géneros en los otros países, se debe a que geográficamente se encuentran en la región tropical y las condiciones ambientales son similares.
En el rango de altura baja, las especies comunes fueron: M. longipes, $P$. psilorhachis, $R$. guatemalensis y $T$. pallida, lo que concuerda el estudio de Vázquez-Negrín et al. (2011); son demandantes de mucha luz solar durante su primera etapa de crecimiento y colonizan claros en la selva (Zarco et al., 2010). En el rango de altura alta estuvo representada por C. brasiliense, D. guianense, G. anomala, $M$. chicle, M. zapota y T. amazonia; las cuales se encuentran en la selva alta perennifolia de México (Pennington \& Sarukhan, 2005). La altura de los árboles de hasta $53 \mathrm{~m}$ en los EA3, 4 y 5 , se le atribuye a la lejanía de la comunidad y el difícil acceso, impidiendo la extracción de madera en donde son empleadas para el uso doméstico. Algunos árboles de mayor altura registran densidad baja, lo que concuerda con los registros de Zarco et al. (2010) y VázquezNegrín et al. (2011), debido a que se convierten en árbol lobo impidiendo la regeneración de la misma especie.

La densidad de los árboles por hectárea no presentó relación entré la altitud, similar a lo obtenido por Brown, Grau, Malizia y Grau (2001) en Argentina. En todos los EA se obtuvieron un mayor número de individuos en la primera categoría diamétrica con una disminución progresiva en las siguientes mostrando 
una distribución de "J" invertida, lo cual coincide con los resultados de Vázquez-Negrín et al. (2011) y López-Toledo et al. (2012), sugiriendo una buena repoblación de las especies arbóreas $\mathrm{y}$ asegurando la permanencia de la selva.

El mayor valor de área basal se encontró en el EA5, por la presencia de T. amazonia, $M$. zapota y $G$. anomala con diámetros de hasta $190 \mathrm{~cm}$ y con dominancia en la selva (Pennington \& Sarukhán, 2005). Encontramos un patrón de a mayor altitud, mayor área basal, tendencia similar con el estudio de Lieberman, Lieberman, Peralta y Hartshorn (1996) en Costa Rica.

Las especies con más altos valores estructurales (IVI e IVF), fueron similares en todo los EA donde no hay perturbación. Lo cual coincide con el estudio de Levy et al. (2006) y Meave et al. (2008), ambos autores argumentan que son parte de la selva alta perennifolia, a excepción de $H$. campechianum y E. gaumeri que son de baja subperennifolia o inundable (Tun-Dzul et al., 2008). Estos valores se deben a que, en altitudes de 400 a $900 \mathrm{~m}$, presentan las mismas condiciones de temperatura y precipitación, que ayuda a la coexistencia de ciertas especies en estas escalas (Cruz, 2013). Debido que el ambiente se modifica en altitudes mayores de $1000 \mathrm{~m}$ y de la mano con la estructura y composición de la vegetación (McAuliffe, 1994).

La diversidad según el índice de $\mathrm{H}^{\prime}$, se presentó con menor valor a mayor altitud, coincide con el valor de $\mathrm{S}$ que mostró dominancia de ciertas especies en cada EA. Esto se atribuye a la altitud que actúa como barrera en la distribución de las especies (Sulca, 2013). Aunque estudio de Ding et al. (2012) en China encontraron mayor diversidad en áreas perturbadas, al proporcionar nichos de regeneración (Chazdon, 2003). La semejanza entre los EA disminuye al aumentar el intervalo de altitud, afirmando la preferencia ambiental particular de cada especie (Araujo-Murakami et al., 2015).

En conclusión, la estructura y diversidad de las especies arbóreas, presentaron mayores cambios a intervalos altitudinales más amplios, y se debe a los requerimientos ecológicos de cada especie, conforme asciende la altitud la temperatura y precipitación disminuye, factores que influyen directamente en la riqueza de especie. La riqueza registrada se debe a la suma de los factores ambientales, provocando que a mayor altitud haya menor diversidad de las especies arbóreas. Las especies inscritas en la NOM-059-SEMARNAT-2019, se deben de estudiar de manera particular, para poder implementar estrategias de conservación.

Los estratos altitudinales estudiados presentaron una repoblación aceptable, esto se debe a la estructura horizontal y vertical de las diferentes especies, que permiten las interacciones entre ellas asegurando la permanencia de la selva, con este resultado se demuestra que la selva del área natural protegida Metzabok es una de las menos afectadas por el disturbio en México.

\section{RESUMEN}

Introducción: Las selvas albergan más de la mitad de las especies del planeta, a pesar de la biodiversidad que poseen son los ambientes más amenazados, principalmente por actividades antrópicas, y su complejidad con relación a la altitud alberga vacíos de información a pesar de los valores que representa. Objetivo: Describir la estructura y diversidad de árboles en un gradiente altitudinal. Métodos: En cinco estratos altitudinales (EA) se establecieron 21 unidades de muestreo (UM) de 20 × $50 \mathrm{~m}$ : seis en el EA1, cinco en EA2, cuatro en EA3, tres en EA4 y 5. La estructura se caracterizó mediante las categorías diamétricas y de altura, y de los índices de valor de importancia (IVI) y forestal (IVF). La diversidad se analizó con los índices de Shannon-Wiener $\left(H^{\prime}\right)$ y Simpson $(S)$, mientras la semejanza florística con el coeficiente de Sørensen (IS). Resultados: Se registraron 209 especies, ocho registradas en la Norma Oficial Mexicana-059-Secretaría de Medio Ambiente y Recursos Naturales-2010 (NOM059-SEMARNAT-2019). Las especies con los mayores IVI e IVF fueron: Haematoxylum campechianum y Manilkara zapota en EA1; Rinorea guatemalensis y Pouteria reticulata en EA2; M. zapota y Brosimum alicastrum en EA3; Guatteria anomala y Pseudolmedia spuria en EA4; y Terminalia amazonia y Pouteria durlandii en EA5. En los cinco EA se identificaron tres rangos de altura y seis de diámetro, el mayor número de individuos se encontró en la primera categoria diamétrica. La diversidad de las especies fue menor a mayor altitud. En los EA 2 y 3 se presentó la mayor semejanza $(I S=58.7$ \%). Conclusión: La estructura y diversidad de las especies arbóreas, presentan mayores 
cambios en intervalos altitudinales más amplios, relacionados con la necesidad ambiental de cada especie.

Palabra clave: elevación; estructura ecológica; área basal; riqueza de especies; semejanza.

\section{REFERENCIAS}

Araujo-Murakami, A., Villarroel, D., Pardo, G., Vos, V.A., Parada, G.A., Arroyo, L., \& Killeen, T. (2015). Diversidad arbórea de los bosques de tierra firme de la Amazonía Boliviana. Kempffiana, 11(1), 1-28.

Basáñez, A.J., Alanís, J.L., \& Badillo, E. (2008). Composición florística y estructura arbórea de la selva mediana subperennifolia del ejido El Remolino, Papantla, Veracruz. Avances en Investigación Agropecuaria, 12(2), 3-21.

Beltrán-Rodríguez, L., Valdez-Hernández, J.I., Luna-Cavazos, M., Romero-Manzanares, A., Pineda-Herrera, E., Maldonado-Almanza, B., Borja-de la Rosa, M.A., \& Blancas-Vázquez, J. (2018). Estructura y diversidad arbórea de bosques tropicales caducifolios secundarios en la Reserva de la Biósfera Sierra de Huautla, Morelos. Revista Mexicana de Biodiversidad, 89(1), 108-122.

Bradford, M.G., Metcalfe, D.J., Ford, A., Liddell, M.J., \& Mckeown, A. (2014). Floristics, stand structure and aboveground biomass of a 25 ha rainforest plot in the wet Tropics of Australia. Journal of Tropical Forest Science, 26(4), 543-553.

Brienen, R.J.W., \& Zuidema, P.A. (2006). Lifetime growth patterns and ages of Bolivian rain forest trees obtained by tree ring analysis. Journal of Ecology, 94, 481-493.

Brown, A.D., Grau, H.R., Malizia, L.R., \& Grau, A (2001). Argentina. In M. Kapelle, \& A.D. Brown (Eds.), Bosques Nublados del Neotrópico (pp. 623659). San José, Costa Rica: INBio.

Carreón-Santos, R.J., \& Valdez-Hernández, J.I. (2014). Estructura y diversidad arbórea de vegetación secundaria derivada de una selva mediana subperennifolia en Quintana Roo. Serie Ciencias Forestales $y$ del Ambiente, 20(1), 119-130. DOI:10.5154/r. rchscfa.2013.06.023

Chazdon, R.L. (2003). Tropical forest recovery: legacies of human impact and natural disturbances. Perspectives in Plant Ecology Evolution and Systematics, 6, 51-71.

Clark, D.A., \& Clark, D.B. (1987). Temporal and environmental patterns of reproduction in Zamia skinneri, a tropical rain forest cycad. Journal of Ecology, $75,135-149$

Comisión Nacional para el Conocimiento y uso de la Biodiversidad (CONABIO). 2008. La diversidad biológica forestal en México. Recuperado de http:// www.conabio.gob.mx/institucion/cooperacion_internacional/doctos/dbf mexico.html.05/02/16

Cruz-Lara, L.E., Lorenzo, C., Soto, L., Naranjo, E., \& Ramírez-Marcial, N. (2004). Diversidad de mamíferos en cafetales y selva mediana de las cañadas de la selva Lacandona, Chiapas, México. Acta Zoológica Mexicana, 20(1), 63-81.

Ding, Y., Zang, R., Liu, S., He, F., \& Letche, S.G. (2012). Recovery of woody plant diversity in tropical rain forests in Southern China after logging and shifting cultivation. Biological Conservation, 145, 225-233.

Dirzo, R., Aguirre, A., \& López, J.C. (2009). Diversidad florística de las selvas húmedas en paisajes antropizados. Investigación Ambiental, 1(1), 17-22.

Durán-Fernández, A., Aguirre-Rivera, J.R., García-Pérez, J., Levy-Tacher, S., \& Nova-Vázquez, J.A.D. (2016). Inventario florístico de la comunidad lacandona de Nahá, Chiapas, México. Botanical Sciences, 94(1), 157-184.

González, E., \& Hamrick, J.L. (2005). Distribution of genetic diversity among disjunct populations of the rare forest understory herb, Trillium reliquum. Heredity, 95, 306-314.

Gourlet-Fleury, S., Beina, D., Fayolle, A., Ouédraogo, D.Y., Mortier, F., Bénédet, F., ... Decocq, G. (2013). Silvicultural disturbance has little impact on tree species diversity in a Central African moist forest. Forest Ecology and Management, 304, 322-332.

Grubb, P. (1977). Control of forest growth and distribution of wet tropical mountains: with special reference to mineral nutrition. Annual Review of Ecology, Evolution, and Systematics, 8, 83-107.

Guariguata, M.R., Cornelius, J.P., Locatelli, B., Forner, C., \& Sánchez-Azofeifa, G.A. (2008). Mitigation needs adaptation: tropical forestry and climate change. Mitigation and Adaptation Strategies for Global Change, 13, 793-808.

Koleff, P., Urquiza-Haas, T., \& Contreras, B. (2012). Prioridades de conservación de los bosques tropicales en México: reflexiones sobre su estado de conservación y manejo. Ecosistemas, 21(2), 6-20.

Levy, T.S.I., Aguirre, R.R.J., García, P.J.D., \& Martínez, R.M.M. (2006). Aspectos florísticos de Lacanhá Chansayab, Selva Lacandona, Chiapas. Acta Botánica Mexicana, 77, 69-98.

Lieberman, D., Lieberman, M., Peralta, R., \& Hartshorn, G.S. (1996). Tropical forest structure and composition on a large-scale altitudinal gradient in Costa Rica. Journal of Ecology, 84(1), 137-152. DOI: $10.2307 / 2261350$ 
López-Toledo, J.F., Valdez-Hernández, J.I., Pérez-Farrera, M.A., \& Cetina-Alcalá, V.M. (2012). Composición y estructura arbórea de un bosque tropical estacionalmente seco en la Reserva de la Biósfera la Sepultura, Chiapas. Revista Mexicana de Ciencias Forestales, 3(2), 43-56.

Mateo, R.G., Felicísimo, A.M., \& Muñoz, J. (2011) Modelos de distribución de especies: Una revisión sintética. Revista Chilena de Historia Natural, 84(2), 217-240. DOI: 10.4067/S0716-078X2011000200008

McAuliffe, J.R. (1994). Landscape evolution, soil formation, and ecological patterns and processes in Sonoran Desert Bajadas. Ecological Monographs, 64, 111-148.

Meave, J.A., Romero-Romero, M.A., Valle-Doménch, A., Rincón-Gutiérrez, A., Martínez, E., \& Ramos, C.H. (2008). Plant diversity assessment in the Yaxchilán Natural Monument, Chiapas, México. Boletín de la Sociedad Botánica de México, 83, 53-76.

Pennington, T.D., \& Sarukhán, J. (2005). Árboles tropicales de México. Manual para la identificación de las principales especies. Ciudad de México, México: UNAM, FCE.

Sagar, R., Raghubanshi, A.S., \& Singh, J.S. (2003). Tree species composition, dispersion and diversity along a disturbance gradient in a dry tropical forest region of India. Forest Ecology and Management, 186, 61-71.

Sánchez-Gutiérrez, F., Valdez-Hernández, J.I., Hernándezde la Rosa, P., \& Beltrán-Rodríguez, L. (2019). Distribución y correlación espacial de especies arbóreas por gradiente altitudinal en la Selva Lacandona,
Chiapas. Revista Mexicana de Ciencias Forestales, 10(54), 74-99. DOI: 10.29298/rmcf.v10i54.590

Sánchez-Gutiérrez, F., Valenzuela-Gómez, A., ValdezHernández, J.I., \& González-González, C.A. (2017). Estructura y diversidad de especies arbóreas del sitio arqueológico "El Mirador", Selva Lacandona, Chiapas. Polibotánica, 44, 79-94. DOI: 10.18387/ polibotanica. 44.6

Sulca, G.L.A. (2013). Distribución de Rogadinae (Hymenoptera: Braconidae) en un gradiente altitudinal en los Andes del sur del Perú. Ecología Aplicada, 12(2), 141-145.

ter Steege, H., Pitman, N.C.A., Killeen, T.J., Laurance, W.F., Pérez, C.A., Guevara, J.E., \& Valenzuela, G.L. (2015). Estimating the global conservation status of more than 15,000 Amazonian tree species. Science Advances, 1, 1-10. DOI:10.1126/sciadv.1500936

Tun-Dzul, F.J., Vester, H., Durán, G.R., \& Schmook, B. (2008). Estructura arbórea y variabilidad temporal del NDVI en los "bajos inundables" de la Península de Yucatán, México. Polibotánica, 25, 69-90.

Vázquez-Negrín, I., Castillo-Acosta, O., Valdez-Hernández, J.I., Zavala-Cruz, J., \& Martínez-Sánchez, J.L. (2011). Estructura y composición florística de la selva alta perennifolia en el ejido Niños Héroes Tenosique, Tabasco, México. Polibotánica, 32, 41-61.

Zarco, E.V.M., Valdez-Hernández, J.I., Ángeles-Pérez, L., \& Castillo-Castillo, O. (2010). Estructura y diversidad de la vegetación arbórea del parque estatal Agua Blanca, Macuspana, Tabasco. Universidad y Ciencia, 26(1), 1-17. 